\title{
ANÁLISE E DESENVOLVIMENTO DE
}

\section{MATERIAIS CERÂMICOS:}

\section{NOVAS PERSPECTNAS}

\section{Organizadores:}

Aluska do Nascimento Simões Braga

Geysivana Késsya Garcia Carvalho Hitalo de Jesus Bezerra da Silva José Rosa de Souza Farias Valdeci Bosco dos Santos Veruska do Nascimento Simões

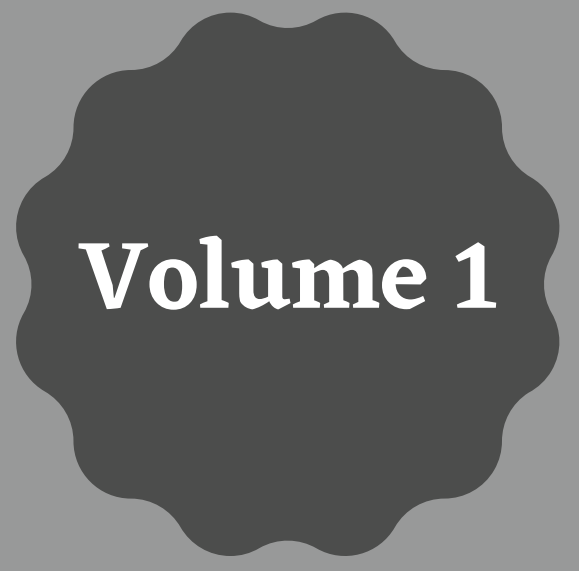
Ycaro Breno Alves de Almeida

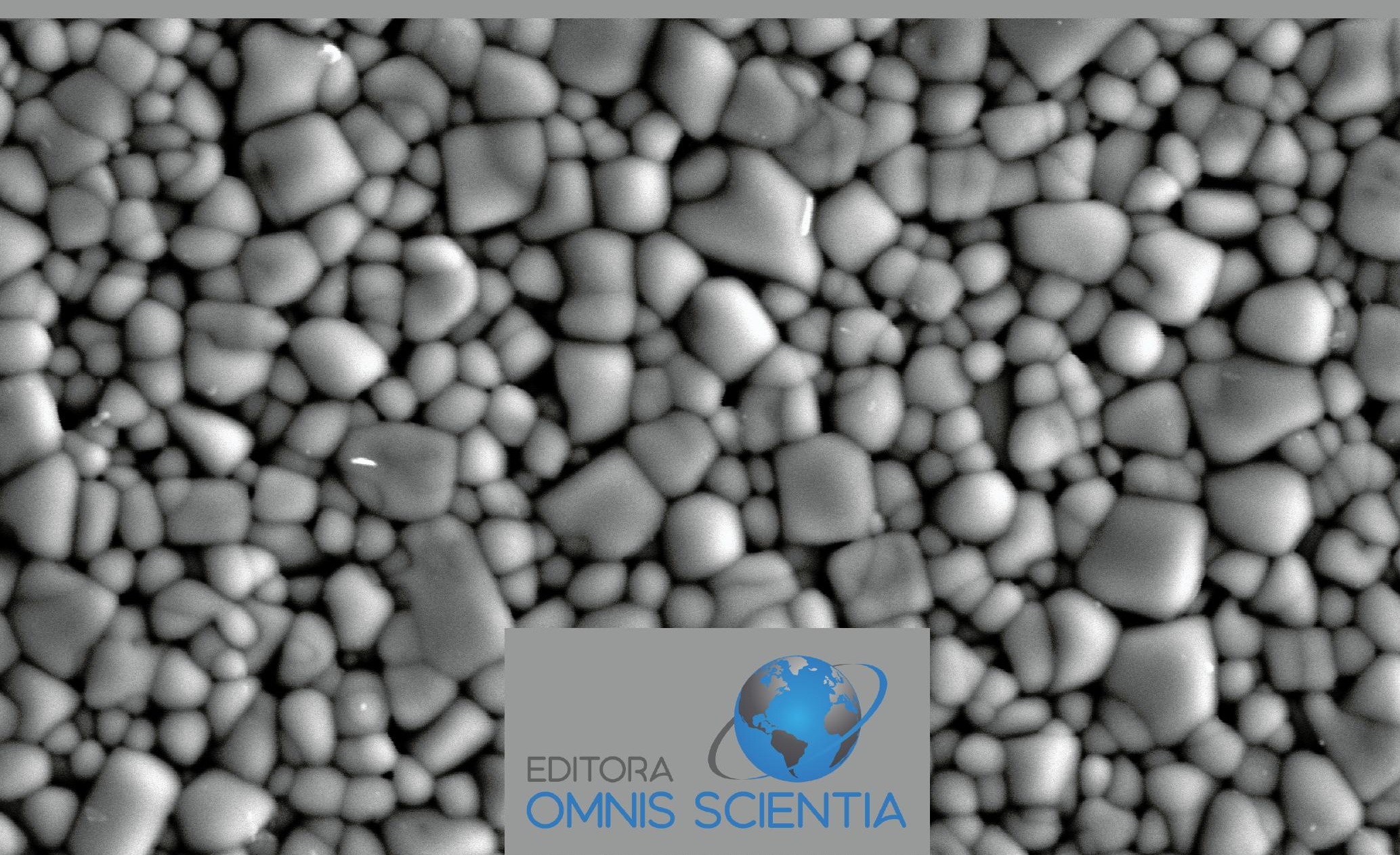




\section{ANÁLISE E DESENVOLVIMENTO DE}

\section{MATERIAIS CERÂMICOS:}

\section{NOVAS PERSPECTNAS}

\section{Organizadores:}

Aluska do Nascimento Simões Braga Geysivana Késsya Garcia Carvalho Hitalo de Jesus Bezerra da Silva José Rosa de Souza Farias Valdeci Bosco dos Santos Veruska do Nascimento Simões

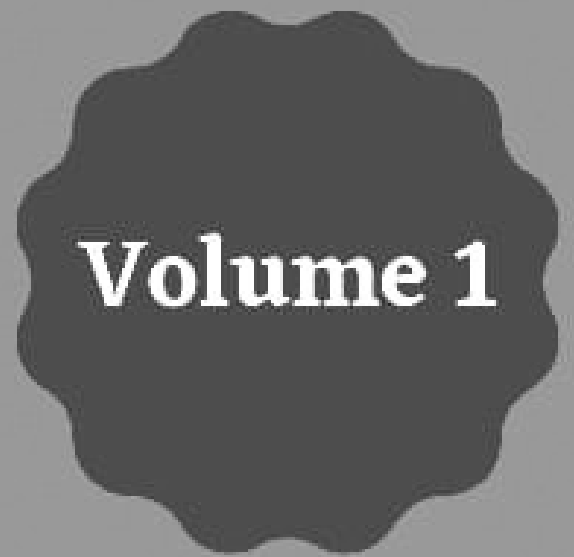
Ycaro Breno Alves de Almeida

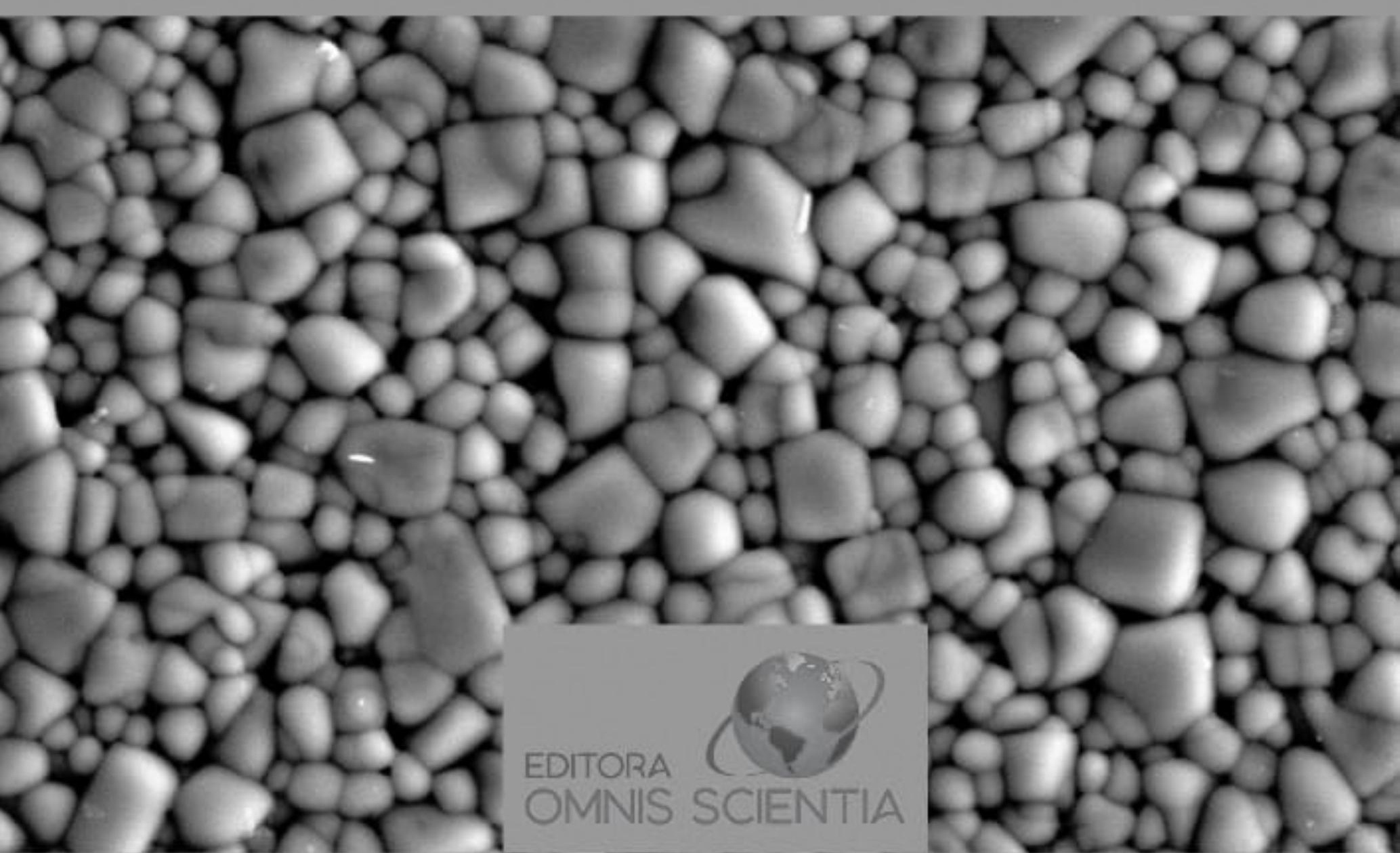


Editora Omnis Scientia

ANÁLISE E DESENVOLVIMENTO DE MATERIAIS CERÂMICOS:

NOVAS PERSPECTIVAS

Volume 1

$1^{\mathrm{a}}$ Edição

TRIUNFO - PE

2022 


\section{Editor-Chefe}

Me. Daniel Luís Viana Cruz

\section{Organizadores}

Aluska do Nascimento Simões Braga

Geysivana Késsya Garcia Carvalho

Hitalo de Jesus Bezerra da Silva

José Rosa de Souza Farias

Valdeci Bosco dos Santos

Veruska do Nascimento Simões

Ycaro Breno Alves de Almeida

\section{Conselho Editorial}

Dr. Cássio Brancaleone

Dr. Marcelo Luiz Bezerra da Silva

Dra. Pauliana Valéria Machado Galvão

Dr. Plínio Pereira Gomes Júnior

Dr. Walter Santos Evangelista Júnior

Dr. Wendel José Teles Pontes

\section{Editores de Área - Engenharias}

Dra. Elba Gomes dos Santos Leal

Dr. Mauro de Paula Moreira

\section{Assistente Editorial}

Thialla Larangeira Amorim

\section{Imagem de Capa}

Os autores

\section{Edição de Arte}

Vileide Vitória Larangeira Amorim

\section{Revisão}

Os autores

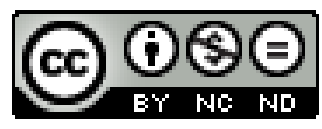

Este trabalho está licenciado com uma Licença Creative Commons - AtribuiçãoNãoComercial-SemDerivações 4.0 Internacional.

O conteúdo abordado nos artigos, seus dados em sua forma, correção e confiabilidade são de responsabilidade exclusiva dos autores. 
Dados Internacionais de Catalogação na Publicação (CIP) (eDOC BRASIL, Belo Horizonte/MG)

A532 Análise e desenvolvimento de materiais cerâmicos [livro eletrônico] : novas perspectivas / Organizadores Aluska do Nascimento Simões Braga... [et al.]. - Triunfo, PE: Omnis Scientia, 2022. 74 p. : il.

Inclui bibliografia

ISBN 978-65-88958-77-3

DOI 10.47094/978-65-88958-77-3

1. Materiais cerâmicos. 2. Cerâmicas tradicionais. 3. Cerâmicas avançadas. 4. Engenharia de materiais. I. Braga, Aluska do Nascimento Simões. II. Carvalho, Geysivana Késsya Garcia. III.Silva, Hitalo de Jesus Bezerra da. IV. Farias, José Rosa de Souza. V.Santos, Valdeci Bosco dos. VI. Simões, Veruska do Nascimento. VII. Almeida, Ycaro Breno Alves de.

CDD 620.11

Elaborado por Maurício Amormino Júnior - CRB6/2422

\author{
Editora Omnis Scientia \\ Triunfo - Pernambuco - Brasil \\ Telefone: +55 (87) 99656-3565 \\ editoraomnisscientia.com.br \\ contato@editoraomnisscientia.com.br
}




\section{PREFÁCIO}

Materiais cerâmicos são uma classe de materiais que abrange uma grande variedade de substâncias naturais e sintéticas, tais como tijolos, revestimentos, pedras, concreto, abrasivos, vidrados para porcelana, isolantes, dielétricos, materiais magnéticos e não-metálicos, refratários para altas temperaturas e muitos outros. Popularmente, o termo cerâmica é utilizado para designar objetos de arte feitos a partir de massas plásticas cerâmicas. Usualmente, os materiais cerâmicos podem ser descritos como compostos inorgânicos formados por elementos metálicos e não metálicos. Visando melhor compreensão dos materiais cerâmicos, costuma-se dividi-los em dois grupos básicos: as cerâmicas tradicionais e as cerâmicas avançadas.

As cerâmicas tradicionais compreendem todos os materiais cerâmicos fabricados a partir de matérias-primas que são encontrados na natureza, como argilas, cerâmicas vermelhas, cerâmicas brancas, entre outros. Os produtos das cerâmicas tradicionais incluem: telhas, tijolos, cimento, revestimentos cerâmicos (pisos e azulejos), louças de mesa, vidros em geral, materiais refratários e isoladores elétricos.

As cerâmicas avançadas, também conhecidas como cerâmicas de alta tecnologia, diferem das tradicionais em virtude do controle mais rígido dos materiais empregados e nas etapas de processamento, o que influi nas propriedades e na microestrutura do material. São compostas por materiais sintéticos de alto grau de pureza, tais como o óxido de alumínio (A12O3), carboneto de silício ( $\mathrm{SiC}$ ), nitreto de silício ( $\mathrm{Si3N} 4)$ e óxido de zircônio ( $\mathrm{ZrO} 2)$, entre outros, preparados por métodos mais sofisticados, envolvendo processos controlados, bem como melhor entendimento a nível microscópico. Os produtos das cerâmicas avançadas incluem: cerâmicas eletrônicas (isolantes e semicondutores), cerâmicas magnéticas (ferritas), cerâmicas ópticas (fibras ópticas, materiais para laser), elementos combustíveis (pastilhas de UO2), etc.

Neste sentido, este livro aborda diferentes pesquisas relacionados aos materiais cerâmicos, apresentando de maneira clara e objetiva resultados de estudos originais, bem como dados e informações pertinentes por meio de revisões bibliográficas acerca dessa área da ciência e engenharia de materiais. 


\section{SUMÁRIO}

CAPÍTULO 1

.10

INFLUÊNCIA DA RAZÃo ÁCIDO CÍTRICO/CÁTIONS METÁLICOS NA SÍNTESE DE MULITA PELO MÉTODO PECHINI

José Rosa de Souza Farias

Slanna Larissa Olimpio Costa

Ycaro Breno Alves de Almeida

Veruska do Nascimento Simões

Geysivana Késsya Garcia Carvalho

Hitalo de Jesus Bezerra da Silva

Érica Karine Alves de Lima

Valdeci Bosco dos Santos

Aluska do Nascimento Simões Braga

DOI: 10.47094/978-65-88958-77-3/10-17

CAPÍTULO 2

EFEITO DO TETRAHIDROFURANO COMO SOLVENTE NA SÍNTESE DE MULITA PELO MÉTODO PECHINI

José Rosa de Souza Farias

Slanna Larissa Olimpio Costa

Ycaro Breno Alves de Almeida

Veruska do Nascimento Simões

Geysivana Késsya Garcia Carvalho

Hitalo de Jesus Bezerra da Silva

Wollia Nayane Araújo Vitoriano

Valdeci Bosco dos Santos

Aluska do Nascimento Simões Braga

DOI: 10.47094/978-65-88958-77-3/18-25 
CAPÍTULO 3

INFLUÊNCIA DE DIFERENTES ÁLCOOIS COMO SOLVENTES NASIINTESE DE MULITA

PELO MÉTODO SOL-GEL

José Rosa de Souza Farias

Slanna Larissa Olimpio Costa

Adryann Millos Santos De Freitas

Ycaro Breno Alves de Almeida

Veruska do Nascimento Simões

Geysivana Késsya Garcia Carvalho

Hitalo de Jesus Bezerra da Silva

Valdeci Bosco dos Santos

Aluska do Nascimento Simões Braga

DOI: 10.47094/978-65-88958-77-3/26-33

CAPÍTULO 4.

AVALIAÇÃO DA CITOTOXICIDADE DE CERÂMICAS BIFÁSICAS DE HAp/ß̧-TCP

Ycaro Breno Alves de Almeida

Slanna Larissa Olimpio Costa

José Rosa de Souza Farias

Diogenes de Moura Junior

Victoria Regia Alves Sales

Ketelly Estefane da Silva Alves

Genivaldo Melo da Rocha

Veruska do Nascimento Simões

Geysivana Késsya Garcia Carvalho

Hitalo de Jesus Bezerra da Silva

Valdeci Bosco dos Santos

Aluska do Nascimento Simões Braga

DOI: 10.47094/978-65-88958-77-3/34-43 
CAPÍTULO 5

SÍNTESE DE HIDROXIAPATITA VIA ROTA PECHINI: UMA BREVE REVISÃO

Ycaro Breno Alves de Almeida

Slanna Larissa Olimpio Costa

José Rosa de Souza Farias

Diogenes de Moura Junior

Victoria Regia Alves Sales

Ketelly Estefane da Silva Alves

Genivaldo Melo da Rocha

Veruska do Nascimento Simões

Geysivana Késsya Garcia Carvalho

Hitalo de Jesus Bezerra da Silva

Valdeci Bosco dos Santos

Aluska do Nascimento Simões Braga

DOI: 10.47094/978-65-88958-77-3/44-59

CAPÍTULO 6 .60

NANOFIBRAS DE MANGANITA DE LANTÂNIO DOPADAS COM ESTRÔNCIO: UMA ANÁLISE BIBLIOMÉTRICA

Érica Karine Alves de Lima

Wollia Nayane Araújo Vitoriano

Hitalo de Jesus Bezerra da Silva

Maria Elayne Rodrigues Alves

Humberto Denys de Almeida Silva

Fernanda da Luz Barbosa

José Rosa de Souza Farias

Ycaro Breno Alves de Almeida

Veruska do Nascimento Simões

Geysivana Késsya Garcia Carvalho

Aluska do Nascimento Simões Braga

Valdeci Bosco dos Santos

DOI: 10.47094/978-65-88958-77-3/60-71 


\section{CAPÍTULO 5}

\section{SÍNTESE DE HIDROXIAPATITA VIA ROTA PECHINI: UMA BREVE REVISÃO}

\section{Ycaro Breno Alves de Almeida ${ }^{1}$;}

Universidade Federal do Piauí (UFPI), Teresina, Piauí.

http://lattes.cnpq.br/4070307554924095

\section{Slanna Larissa Olimpio Costa ${ }^{2}$;}

Instituto Federal de Educação Ciência e Tecnologia do Piauí (IFPI), Teresina, Piauí.

http://lattes.cnpq.br/5896223773412182

José Rosa de Souza Farias ${ }^{3}$;

Universidade Federal do Piauí (UFPI), Teresina, Piauí.

http://lattes.cnpq.br/9404467330103347

\section{Diogenes de Moura Junior ${ }^{4}$;}

Universidade Federal do Piauí (UFPI), Teresina, Piauí.

http://lattes.cnpq.br/5791161214413731

\section{Victoria Regia Alves Sales 5 ;}

Universidade Federal do Piauí (UFPI), Teresina, Piauí.

http://lattes.cnpq.br/4380086092580232

Ketelly Estefane da Silva Alves';

Universidade Federal do Piauí (UFPI), Teresina, Piauí.

http://lattes.cnpq.br/0511638279355579

\section{Genivaldo Melo da Rocha ${ }^{7}$;}

Universidade Federal do Piauí (UFPI), Teresina, Piauí.

http://lattes.cnpq.br/4165901488191140

Veruska do Nascimento Simões ${ }^{8}$;

Universidade Federal do Rio Grande do Norte (UFRN), Natal, Rio Grande do Norte.

http://lattes.cnpq.br/1149592827133122

\section{Geysivana Késsya Garcia Carvalho";}

Universidade Federal do Piauí (UFPI), Teresina, Piauí.

http://lattes.cnpq.br/0242153749540840 


\title{
Hitalo de Jesus Bezerra da Silva ${ }^{10}$;
}

Universidade Federal de Pernambuco (UFPE), Recife, Pernambuco.

http://lattes.cnpq.br/4510351692039237

\section{Valdeci Bosco dos Santos ${ }^{11}$;}

Universidade Federal do Piauí (UFPI), Teresina, Piauí.

Instituto Federal de Educação, Ciência e Tecnologia do Piauí (IFPI), Teresina, Piauí.

http://lattes.cnpq.br/0011700686113389

\author{
Aluska do Nascimento Simões Braga ${ }^{12}$. \\ Universidade Federal do Piauí (UFPI), Teresina, Piauí. \\ Instituto Federal de Educação, Ciência e Tecnologia do Piauí (IFPI), Teresina, Piauí. \\ $\underline{\text { http://lattes.cnpq.br/2429557575387821 }}$
}

RESUMO: Compondo um grupo de materiais destinados a interagir com o ambiente fisiológicos do organismo humano, os biomateriais podem desempenhar funções de tecidos e órgãos promovendo uma melhora na qualidade de vida dos pacientes. As biocerâmicas se destacam frente aos outros materiais utilizados com esta finalidade, principalmente em decorrência da biocompatibilidade intrínseca de suas composições. A hidroxiapatita (HAp) é uma apatita extremamente versátil, membro da família de fosfatos de cálcio, que é extremamente visada para aplicações biológicas devido a propriedades como biocompatibilidade e bioatividade inerentes de sua composição. Sendo termodinamicamente estável em seu estado cristalino no fluido corporal, podendo integrar-se ao osso sem causar toxicidade local ou sistêmica, sendo extensamente utilizada na área ortopédica e ortodôntica. O interesse nesse material é notório, uma vez que muitos métodos químicos viabilizam sua obtenção. O método Pechini é uma alternativa viável, no entanto pouco discutida na literatura para a obtenção da Hap. Com isso, este trabalho tem como objetivo realizar uma revisão acerca da hidroxiapatita e seus métodos químicos de obtenção, focando na sua obtenção pelo método Pechini. Observou-se que as condições de síntese afetam fortemente o produto final. Onde, a pureza do material está intimamente ligado à relação $\mathrm{Ca} / \mathrm{P}$ estabelecida. A relação ácido cítrico/cátions metálicos, o solvente, o agente gelificante e do agente complexante utilizados na síntese também impactam na cerâmica produzida.

PALAVRAS-CHAVE: Síntese. Método Pechini. Hidroxiapatita 
ABSTRACT: Composing a group of materials designed to interact with the physiological environment of the human body, biomaterials can perform tissue and organ functions promoting an improvement in the quality of life of patients. Bioceramics stand out compared to other materials used for this purpose, mainly due to the intrinsic biocompatibility of their compositions. Hydroxyapatite (HAp) is an extremely versatile apatite, a member of the calcium phosphate family, which is highly targeted for biological applications due to properties such as biocompatibility and bioactivity inherent to its composition. Being thermodynamically stable in its crystalline state in the body fluid, it can integrate with the bone without causing local or systemic toxicity, being extensively used in the orthopedic and orthodontic areas. The interest in this material is notorious, since many chemical methods make it possible to obtain it. The Pechini method is a viable alternative, however little discussed in the literature for obtaining Hap. Thus, this work aims to carry out a review about hydroxyapatite and its chemical methods of obtaining, focusing on its obtainment by the Pechini method. It was observed that the synthesis conditions strongly affect the final product. Where, the purity of the material is closely linked to the established $\mathrm{Ca} / \mathrm{P}$ ratio. The citric acid/metal cations ratio, the solvent, the gelling agent and the complexing agent used in the synthesis also impact the ceramic produced.

KEY-WORDS: Synthesis. Pechini method. Hydroxyapatite.

\section{INTRODUÇÃO}

Biomateriais são comumente definidos como uma substância não viável que interage com o biossistema, sendo usados para substituir ou melhorar a função dos tecidos e órgãos que foram danificados ou sofreram degeneração, promovendo uma melhora na qualidade de vida dos pacientes (Raval et al., 2019). Na última década, houve um progresso significativo em direção ao desenvolvimento de biomateriais para serem utilizados na fabricação de dispositivos inovadores para uma ampla variedade de aplicações biomédicas (Guarivo et al., 2020). Tal fato ocorreu devido ao mercado de bioimplantes está aumentando exponencialmente com o crescimento da população em envelhecimento, mudanças no estilo de vida, avanços tecnológicos em bioengenharia e aumento da conscientização sobre implantes cosméticos (Kumar et al., 2020).

Dentre as classes de materiais utilizados para aplicações biológicas destacam-se as biocerâmicas. As biocerâmicas podem ser de origem natural ou sintética, compondo uma classe de cerâmicas inorgânicas e não metálicas usadas para reparo e regeneração de partes doentes e danificadas do sistema musculoesquelético e anomalias periodontais (Pina et al., 2018). No que diz respeito à biocerâmica, estabilidade térmica, resistência mecânica, biocompatibilidade e suas semelhanças com os tecidos ósseos são os fatores que influenciam sua implementação em diferentes aplicações (Naik, 2019). Assim as biocerâmicas mais demandadas para a prática clínica no último terço do século XX podem ser classificados em dois grupos principais: cerâmica bioativa, como fosfatos de cálcio, vidros e cerâmicas de vidro e cerâmica inerte, como alumina, zircônia e carbono (Vallet-regi et al., 
2008). Sendo extensamente usada em vários tratamentos, como obturações dentárias, tratamentos periodontais, substituições de articulações e membros, reconstrução craniomaxilofacial, artérias artificiais e substitutos da pele, lentes de contato e substitutos ósseos (Naik, 2019).

As biocerâmicas de fosfato de cálcio (CaP) compõem um grupo muito importante de biomateriais, que surgem como materiais reabsorvíveis e bioativos. $\mathrm{O}$ uso de vários materiais de fosfato de cálcio para reparo ósseo, aumento, substituição e como revestimentos em implantes metálicos ganhou aceitação clínica em muitas aplicações odontológicas e médicas (Pierre et al., 2019; Rustom et al., 2019; Shyong et al., 2018; Konermann et al., 2014). A hidroxiapatita (HAp) é o maior representante desta familia de apatitas. Isso ocorre, pois dentes e ossos, possuem na hidroxiapatita seu maior constituinte inorgânico (Pekounov et al., 2009). Dessa forma, devido à similaridade composicional, a HAp pode integrar-se ao osso sem causar toxicidade local ou sistêmica, inflamação ou resposta de corpo estranho (Mohd Pu'ad et al., 2019). Com isso, o interesse em sua obtenção é instigada, e uma gama de rotas laboratoriais foram desenvolvidas com o intuito de obter a HAp sintética. O método Pechini é uma rota bastante atrativa, entretanto pouco visualizada, pois possui discretas discussões na literatura voltada para a obtenção da HAp.

Dessa forma, este trabalho tem como objetivo fazer uma revisão da literatura sobre a hidroxiapatita e sua síntese pela rota Pechini. Assim, espera-se divulgar o conhecimento desta abordagem para a síntese desse material em pesquisas científicas e contribuir para o desenvolvimento tecnológico.

\section{METODOLOGIA}

Para a realização desta revisão foi realizada uma busca na literatura, por meio de pesquisa em banco de dados. As buscas foram utilizadas nas plataformas do Sciencedirect e do Google acadêmico. Sendo utilizados os seguintes termos nas mesmas: "Hydroxyapatite" and "Pechini Method". Os resultados encontrados foram refinados e citados no trabalho.

\section{REFERENCIAL TEÓRICO}

\section{Hidroxiapatita}

Os fosfatos de cálcio são minerais comuns na terra (Epple, 2018). Apresentam como principais características: semelhança com a fase mineral de ossos; dentes e tecidos calcificados; excelente biocompatibilidade; bioatividade; ausência de toxicidade local ou sistêmica; ausência de resposta a corpo estranho ou inflamações; aparente habilidade em se ligar ao tecido hospedeiro; taxas de degradação variáveis; osteocondutividade (Gomes et al., 2012). São convenientemente classificados, por meio da razão molar entre os átomos de cálcio e fósforo $(\mathrm{Ca} / \mathrm{P})$, a qual varia de 0,5 a 2,0. Onde de forma geral, quanto maior a razão $\mathrm{Ca} / \mathrm{P}$, menor será a solubilidade (Guastaldi e Aparecida, 2010). O membro mais amplamente usado da família de fosfato de cálcio é a hidroxiapatita (HAp) (Supová, 2015). 
A hidroxiapatita (HAp) $\left(\mathrm{Ca}_{10}\left(\mathrm{PO}_{4}\right)_{6}(\mathrm{OH})_{2}\right)$ é um mineral essencial bem conhecido do osso humano composto por $70 \%$ de compostos inorgânicos de fosfato de cálcio da apatita e $30 \%$ de materiais orgânicos de células de colágeno e medula óssea (Santhiyavimal et al., 2020). A fórmula química e a estrutura cristalina da HAp são bastante semelhantes às do constituinte inorgânico principal dos ossos e dentes humanos (Luo et al., 2020), apresentando relação Ca/P igual a 1,67 com uma densidade de 3,16 g/cm3 (Yadav et al., 2020). A estrutura cristalina da célula unitária pode ser monoclínica ou hexagonal. No entanto, este último está envolvido na formação de tecidos duros, que é a forma mais frequente e pertence ao grupo espacial $\mathrm{P} 63 / \mathrm{m}$ com parâmetros de rede $\mathrm{a}=\mathrm{b}=9.432 \AA \mathrm{e} \mathrm{c}=6.881 \AA$ (Chen et al., 2018). A Figura 1 apresenta a estrutura da hidroxiapatita.

Figura 1: Estrutura cristalina da hidroxiapatita

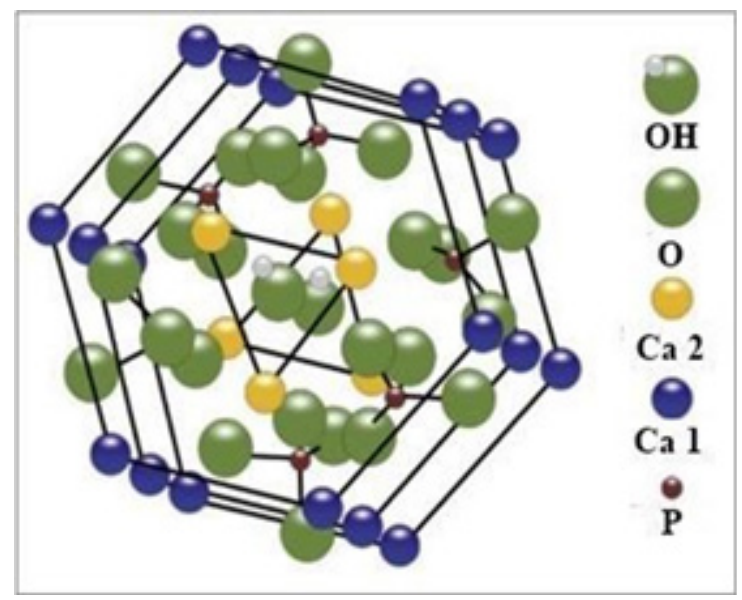

Fonte: Ibrahim et al., 2020

A HAp inclui dois tipos de cátions de cálcio, chamados $\mathrm{Ca}_{1}$ e $\mathrm{Ca}_{2}$. Os átomos de cálcio $\mathrm{Ca}_{1}$ estão localizados nas bordas de uma célula unitária hexagonal, perto do $\mathrm{Ca}$ em torno grupos $-\mathrm{PO}_{4}$ capaz de se ligar com os tecidos vivos e ósseas associadas com ossos fortes, enquanto os átomos de cálcio $\mathrm{Ca}_{2}$ formam triângulos equilaterais com a coluna de grupos hidroxila estruturais no meio (Bootchanont et al., 2017). Os grupos fosfato encontram-se na parte externa do arranjo hexagonal, e sua estrutura tetraédrica exclusiva torna a hidroxiapatita estável (Chen et al., 2018).

Mesmo sob os fatores termodinâmicos e fisiológicos de $\mathrm{pH}$, temperatura e constituição de fluidos corporais, afirma ser um composto muito seguro. Essa natureza biocompatível e bioabsorvível da hidroxiapatita a transforma em um compost elegível para aplicações biomédicas (Shaly et al., 2020). No entanto, a hidroxiapatita nos ossos e dentes humanos não é estequiométrica ou pura, mas contém vários elementos, incluindo carbonatos de $\mathrm{CO}_{3}$ (Benataya et al., 2020). Os íons carbonato na hidroxiapatita carbonatada (CHA) do tipo A substituem os íons hidroxila na rede HAp, enquanto nos íons do fosfato CHA do tipo B são substituídos pelos íons carbonato. Os íons carbonato são predominantemente apresentados como carbonatos do tipo B em minerais ósseos naturais (Kong et al., 2019). Predominantemente, a preparação do tipo $\mathrm{A}\left(\mathrm{Ca}_{10}\left(\mathrm{PO}_{4}\right)_{6} \mathrm{OH}_{2-2 \mathrm{x}}\left(\mathrm{CO}_{3}\right) \mathrm{x}\right)$ geralmente ocorre em uma atmosfera seca de $\mathrm{CO}_{2}$ em alta temperatura $\left(800-1000^{\circ} \mathrm{C}\right)$ na qual os locais de $\mathrm{OH}$ estavam 
substituídos por $\mathrm{CO}_{3}$. Por outro lado, o tipo B é geralmente obtido por métodos úmidos, usando reações de precipitação ou hidrólise a baixa temperatura $\left(20-120^{\circ} \mathrm{C}\right)$ (Benataya et al., 2020). A fase HAp encontrada no osso natural contém aproximadamente 7,4\% em peso de carbonato e o CHA tipo B é a substituição preferida. Estudos demonstraram que o CHA tipo B está associado a uma melhor biocompatibilidade, maior osteocondução e melhor proliferação celular em comparação com HAp (Senra et al., 2020).

\section{DOPAGEM DA HIDROXIAPATITA}

Embora a hidroxiapatita pura tenha sido usada com sucesso em certos casos, ela ainda é limitada por sua fragilidade, instabilidade e adsorvibilidade (Chen et al., 2018). A limitada resistência bacteriana é um outro empecilho de grande impacto, pois as infecções bacterianas são a principal causa de falha do implante (Riaz et al., 2018). Com o intuito de contornar tais obstáculos alguns autores já se concentraram na modificação da hidroxiapatita. A dopagem com íons, é uma das principais tecnologias de modificações, isso ocorre devido a uma importante característica da estrutura da hidroxiapatita, que permite a incorporação de uma ampla gama de íons estranhos (Ciobanu e Harja, 2019).

A incorporação de íons na estrutura da HAp resultará em algumas mudanças estruturais, que podem afetar a estrutura do cristal, a cristalinidade, a carga superficial, a solubilidade e outras propriedades vitais, levando a grandes mudanças no desempenho biológico após a implantação (Supová, 2015). Além disso, para uso biológico, isso também pode alterar as propriedades da superfície dos materiais, como propriedades de adsorção, essenciais na interação do material com íons e moléculas no corpo, biocompatibilidade, bioatividade e antimicrobilidade (Ullah et al., 2020).

Magnésio $\left(\mathrm{Mg}^{2+}\right)$, zinco $\left(\mathrm{Zn}^{2+}\right)$, manganês $\left(\mathrm{Mn}^{2+}\right)$, estrôncio $\left(\mathrm{Sr}^{2+}\right)$, cobre $\left(\mathrm{Cu}^{2+}\right)$, cério $\left(\mathrm{Ce}^{3+}\right)$, $\operatorname{prata}\left(\mathrm{Ag}^{+}\right)$, flúor( $\left.\mathrm{F}^{-}\right)$são alguns substitutos iônicos comumente utilizados como dopantes conferindo a HAp diferentes atividades biológicas (Cao et al., 2020; Wu et al., 2020; Zhou et al., 2020; Mahmood et al., 2020). Esses dopantes podem substituir parcialmente os cátions $\mathrm{Ca}^{2+}$, bem como os ânions $\mathrm{OH}^{-}$ e $\mathrm{PO}_{4}^{3-}$ (Mahmood et al., 2020).

Estudos in vivo demonstraram que a presença de Magnésio $\left(\mathrm{Mg}^{2+}\right)$ na HAp melhora o comportamento celular, adesão a tecidos duros sintéticos, proliferação e ativação metabólica (Montoya- Cisneros et al., 2017). Os íons zinco $\left(\mathrm{Zn}^{2+}\right)$ podem inibir efetivamente bactérias grampositivas e gram-negativas. Além disso, o $\mathrm{Zn}^{2+}$ também possui excelente atividade osteogênica, podendo promover a atividade osteoblástica e a mineralização óssea e inibir a atividade osteoclástica (Zhou et al., 2020). A presença de aditivos de manganês $\left(\mathrm{Mn}^{2+}\right)$ na estrutura cristalina da HAp influencia a aderência das células ósseas ao material do implante, sua disseminação e viabilidade. Isso ocorre devido a alterações conformacionais das integrinas, ou seja, um grupo de receptores responsáveis pela ativação dos processos de adesão celular, causados pelo manganês (Paluszkiewicz et al., 2010). Estrôncio $\left(\mathrm{Sr}^{2+}\right)$ desempenha um grande papel na cárie dentária, remineralização óssea, inibição da reabsorção do tecido ósseo e tratamento da osteoporose óssea (Mahmood et al., 2020). $\mathrm{O}$ cobre $\left(\mathrm{Cu}^{2+}\right)$ atraiu um interesse particular, pois parece estimular a infiltração de vasos sanguíneos 
em implantes que acelera o processo de cicatrização. Além disso, a incorporação de íons de cobre na matriz HAp poderia possivelmente melhorar a integridade mecânica e a capacidade antibacteriana dos revestimentos para aplicação biomédica (Unabia et al., 2018). Materiais que contêm prata têm propriedades antibacterianas, pois a prata pode impedir o crescimento de biofilme e a colonização bacteriana nas várias superfícies (Dubnika e Zalite, 2014). Propriedades antibacterianas são encontradas na hidroxiapatita contendo cério $\left(\mathrm{Ce}^{3+}\right)$, isso ocorre devido à sua ação antibacteriana, cuja ação biocida ocorre em baixas concentrações de cério com efeito prolongado contribuindo para prevenir infecções pós-operatórias principalmente em procedimentos cirúrgicos de implantes ou próteses (Santos et al., 2020). O íon fluoreto ( $\left.\mathrm{F}^{-}\right)$pode promover a nucleação do HAp e substituir $\mathrm{OH}$ no HAp para formar fluorapatita, que possui boa rigidez, alto módulo de elasticidade e dureza, pode ainda estimular a diferenciação de células-tronco mesenquimais em osteoblastos. (Cao et al., 2020), além disso pode reduzir a formação de cárie em ambientes contaminados por bactérias e promover a mineralização e cristalização de fosfatos de cálcio na formação óssea (Trewin et al., 2013).

Martinez-Garcia et al. (2020) realizaram a obtenção de compósitos Ag-Cu / HAp. Onde a hidroxiapatita utilizada foi obtida utilizando a rota Pechini, e a dopagem com prata $\left(\mathrm{Ag}^{+}\right)$foi realizada pelo mesmo meio. Os compósitos Ag-HAp exibiram maior viabilidade celular em comparação com os compósitos $\mathrm{Cu}-\mathrm{HAp}$, que exibiram menor porcentagem de hemolítica. A atividade antibacteriana exibida pela maioria dos compósitos foi mais eficaz, embora tivessem concentrações menores (\%) de prata e cobre do que em estudos anteriores.

Atualmente, na área biotecnológica, grande atenção tem sido focada nos elementos de terras raras, íons como $\mathrm{Ce}^{3+}, \mathrm{Eu}^{3+}, \mathrm{Tb}^{3+}$ e $\mathrm{Gd}^{3+}$. A HAp dopada com estes lantanídeos pode superar a baixa intensidade de fluorescência e a baixa estabilidade de luz dos corantes fluorescentes tradicionais, podendo ser adequada para uso na bioimagem de células de mamíferos (Mahmood et al., 2020; Wang et al., 2020).

\section{Síntese da Hidroxiapatita}

A HAp pode ser sintetizada por uma variedade de técnicas que podem ser amplamente agrupadas em seis conjuntos de métodos: i) métodos secos: envolvem reações de estado sólido e mecânico-químicas ii) métodos úmidos: baseados em precipitação química a baixa temperatura, co-precipitação, rota sol-gel e hidrólise; iii) métodos hidrotérmicos: use soluções aquosas de alta temperatura e alta tensão, como hidrotérmica, emulsão e microemulsão e sonoquímica; iv) processos de alta temperatura: incluem combustão e pirólise; v) síntese baseada em fontes biogênicas: pode ser extraída de espinhas de peixe, conchas, cascas de ovos, ossos de bovinos, na presença de biomoléculas ou biomembranas; e vi) combinação dos métodos mencionados (Vahdat et al., 2020).

A abordagem sol-gel é uma tecnologia ideal para a fabricação de biomateriais, devido à sua capacidade de manipular a estrutura de biomateriais no nível molecular e de realizar a reação à baixa temperatura. $\mathrm{O}$ tempo de envelhecimento é necessário para concluir a reação entre precursores moleculares, e o tratamento térmico apropriado pode acelerar a formação da fase de apatita (Zhang et al., 2014). As principais reações do processo sol-gel - química da hidrólise e condensação - fornecem 
óxidos inorgânicos ou materiais híbridos usando compostos organometálicos, alcóxidos, alcóxidos de metais, óxidos inorgânicos ou sais de metais. Onde, a taxa de é limitada pela reatividade dos precursores (Startek et al., 2020). Especificamente, os materiais que contêm elementos ativos são escolhidos como precursores. O combustível é misturado uniformemente na fase líquida e, em seguida, são realizadas reações de hidrólise e condensação para formar um sistema transparente estável, conhecido como "sol". Depois disso, o "sol" pode ser ainda mais ligado através da condensação para formar um "gel" ajustando parâmetros como valor de pH e temperatura e, finalmente, formar uma estrutura de rede tridimensional (Wang et al., 2020).

Este método oferece uma mistura molecular de cálcio e fósforo, o que melhora a homogeneidade química dos materiais resultantes. Além disso, a alta reatividade dos pós sol-gel resulta na diminuição das temperaturas de calcinação e sinterização (Rajabi-Zamani et al., 2008). Devido à possibilidade de introdução de muitos substratos diferentes na síntese sol-gel e em várias condições do processo, é possível obter diferentes produtos finais em termos de: composição, estruturas, funcionalidade e formas (Startek et al., 2020). Normalmente a preparação de HAp pelo processo sol-gel resulta em uma microestrutura de grão fino contendo uma mistura de cristais nano-submicrônicos. Esses cristais podem ser mais bem aceitos pelo tecido hospedeiro (Fathi e Hanifi, 2007).

\section{Método Pechini}

De acordo com Kakihana (Kakihana, 1996), a abordagem sol-gel apresenta certas nuance; o sol-gel com gel polimérico orgânico se trata de uma destas facetas. O método Pechini, também conhecido como método dos percursores poliméricos se encontra nessa categoria. Desenvolvido em 1967, primordialmente para a síntese de titanatos e niobatos por Maggio Pechini (Pechini, 1967), tornou-se uma técnica sintética atraente para a preparação de vários óxidos inorgânicos (Winck et al., 2017). Excelente controle da estequiometria, tamanho de partícula, capacidade de preparar pós cerâmicos nanoestruturados multicomponentes, boa homogeneidade através da mistura no nível molecular em solução e baixas temperaturas de calcinação necessárias para a decomposição do gel nos materiais de óxido de metal são benefícios que direcionam bastante atenção para este processamento (Longman-Estarki et al., 2017).

A rota Pechini é basicamente um processo de redução eletroquímica envolvendo nitratos metálicos (como oxidantes), ácido cítrico (como combustível) e etileno glicol (como agente de polimerização), que envolve a hidrólise de precursores moleculares constituintes e subsequentemente policondensação ao gel como material (Pubby et al., 2018). Este método baseia-se na quelação catiônica por ácido cítrico e na poliesterificação entre ácido cítrico e etileno glicol, o que leva à formação de um intermediário de resina policatiônica. Este intermediário de resina resultante contém átomos de metal ligados através de oxigênio a radicais orgânicos que compreendem a rede reticulada e, assim, interconectam as espécies reagentes (Nguyen et al., 2019). A policondensação do etileno glicol e ácido cítrico começa acima de $100{ }^{\circ} \mathrm{C}$, resultando na formação de gel de citrato de polímero. Quando a temperatura de aquecimento excede $400^{\circ} \mathrm{C}$, começam a oxidação e pirólise da matriz polimérica, que levam à formação de óxido amorfo. O aquecimento adicional resulta na formação do 
material necessário com um alto grau de homogeneidade e dispersão (Ramirez et al., 2017). Assim, promove uma dispersão homogênea de metais em toda a rede de polímeros devido ao seu crescimento controlado. Posteriormente, a lenta decomposição da matriz polimérica permite maior controle sobre a formação do material (Santos et al., 2020). A figura 2 apresenta um resumo esquemático do processo Pechini.

Figura 2: Representação esquemática do processo Pechin

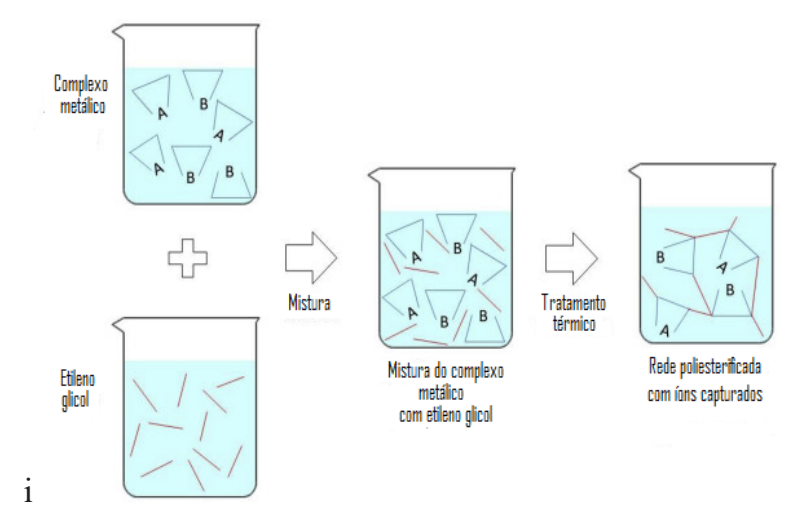

Fonte: Kumar et al., 2019

Entretanto, mesmo se tratando de um método bastante promissor, esta rota é pouco reproduzida e discutida para a síntese de HAp. A seguir é apresentado todos os artigos resultantes da busca relacionada a produção de hidroxiapatita através do método dos percursores poliméricos.

Peña e Vallet-regí (2003) propuseram a aplicação da Técnica de Mistura Líquida, que se baseia na patente Pechini para preparação de fosfatos de cálcio. O processo utilizou os sais metálicos $\left(\mathrm{CaNO}_{3} \cdot 4 \mathrm{H}_{2} \mathrm{O}\right.$ e $\left.\mathrm{H}_{2} \mathrm{NH}_{4} \mathrm{PO}_{4}\right)$ como percursores, obedecendo uma razão molar de 1:1 com CA. Onde as quantidades empregadas para preparar as diferentes composições que abrangiam desde o beta TCP (relação $\mathrm{CaP}=1,5$ ) até a hidroxiapatita (relação $\mathrm{CaP}=1,67$ ). Dessa forma, através deste método eles conseguiram obter hidroxiapatita monofásica, $\beta$-TCP e $\alpha$-TCP e também materiais bifásicos cujo teor em $\beta$-TCP e HAp pode ser previsto com precisão a partir da razão $\mathrm{Ca} / \mathrm{P}$ nas soluções precursoras.

Omori et al. (2014) relatam um método Pechini modificado que envolve um $\mathrm{Ca} / \mathrm{P}$ molar inicial > 1,67 para a fabricação de nanocristais HAp dispersíveis. Utilizaram os sais metálicos $\left(\mathrm{CaNO}_{3} \cdot 4 \mathrm{H}_{2} \mathrm{O}\right.$ e NH4H2PO4) como materiais de partida e obedeceram uma razão molar de 1:1 com CA. Assim demonstraram que o método Pechini em condições não estequiométricas leva à formação de uma fase de cristal binária (HAp e CaO). Também demonstraram que nanocristais de HAp biocerâmicos altamente cristalinos que podem ser dispersos em meio líquido, principalmente na forma de nanopartículas, foram obtidos após a dissolução de uma fase da matriz (isto é, CaO) nos casos em que a quantidade do componente da matriz formado foi suficientemente maior do que a quantidade do componente nanocristal formado.

Carvalho et al. (2020) avaliaram as mudanças estruturais e morfológicas provocadas pela razão ácido cítrico /cátions metálicos na hidroxiapatita produzida através do método Pechini modificado. 
Nitrato de cálcio e Fosfato de amônio dibásico foram os precursores utilizados e estiveram sob diferentes relações ácido cítrico /cátions metálicos (1:1, 2:1, 3:1, 4:1 e 5:1). A relação ácido cítrico/ cátions metálicos $(\mathrm{AC} / \mathrm{CM})$ influenciou na formação das fases, onde para todas as proporções de ácido cítrico/cátions metálicos estudadas foi obtida uma cerâmica bifásica composta por HAp e $\beta$ -TCP, porém com porcentagem de fases diferentes. A relação 4:1 de AC/CM foi o parâmetro com melhores resultados.

Farias et al. (2020) avaliaram a relação ácido cítrico/cátions metálicos, no entanto sob uma nova circunstâncias. Nitrato de cálcio e fosfato de amônio dibásico sob as relações 1:1, 3:1 e 5:1, foram preparadas em um novo solvente, álcool etílico PA. As sínteses resultaram na formação de uma cerâmica bifásica, com segunda fase de $\beta$-TCP. No entanto, não houve mudanças significativas nas quantificações de fases nas variações de AC/CM estudadas.

Swamiappan (2016) obteve a hidroxiapatita utilizando nitrato de cálcio e o hidrogenofosfato de diamônio como fontes de cálcio e fósforo, respectivamente. Avaliando a influência do agente gelificante (etilenoglicol) e do agente complexante (ácido cítrico) na pureza do produto. O produto resultante foi a hidroxiapatita substituída com carbonato e os íons carbonato substituídos reduzem a estabilidade térmica do biomaterial preparado.

Por meio desta breve revisão, observa-se a forte influência dos parâmetros de síntese frente às condições de síntese da Hap pelo método Pechini, afetando em larga escala o produto resultante. E, como esta promissora rota de síntese ainda precisa ser investigada na obtenção de cerâmicas de fosfatos de cálcio, como a hidroxiapatita.

\section{CONCLUSÃO}

Através desta breve revisão nota-se a importância da hidroxiapatita frente às suas aplicações, o que desperta o grande interesse nesse material, onde em decorrência disso, uma vasta gama de meios são responsáveis por sua obtenção. O método Pechini apresentou-se como um candidato bastante promissor, que apresenta uma série de vantagens. E no que tange a síntese de hidroxiapatita por Pechini, as condições de síntese afetam fortemente o produto final. Onde, a pureza do material está intimamente ligado relação $\mathrm{Ca} / \mathrm{P}$ estabelecida, em relações inferiores hidroxiapatita monofásica, $\beta$-TCP e $\alpha$-TCP e também materiais bifásicos podem ser obtidos, enquanto em teores superiores, as fases Hap e $\mathrm{CaO}$ podem coexistir. A relação ácido cítrico/cátions metálicos, o solvente, o agente gelificante e do agente complexante utilizados na síntese também podem influenciar na cerâmica produzida. Percebeu-se também que apesar da relevância do método em questão, na literatura mundial existem pouquissimos trabalhos que estudaram a obtenção da HAp pelo método Pechini.

\section{DECLARAÇÃO DE INTERESSES}

Nós, autores deste artigo, declaramos que não possuímos conflitos de interesses de ordem financeira, comercial, político, acadêmico e pessoal. 


\section{REFERÊNCIAS}

BENATAYA, K; LAKRAT, M; ELANSARI, L. L; MEJDOUBI, E. Synthesis of B-type carbonated hydroxyapatite by a new dissolution-precipitation method. Materials Today: Proceedings, Volume 31, Supplement 1, 2020 , páginas S83-S88.

BOOTCHANONT, A; SAILUAM, W; SUTIKULSOMBAT, S; TEMPROM, L; CHANLEK, N; KIDKHUNTHOD, P; SUWANNA, P; YIMNIRUN, R. Synchrotron X-ray Absorption Spectroscopy study of local structure in strontium-doped hydroxyapatite. Ceramics International, 43, 14, 11023-11027, 2017.

CAO, J; LIAN, R; JIANG, X. Magnesium and fluoride doped hydroxyapatite coatings grown by pulsed laser deposition for promoting titanium implant cytocompatibility. Applied Surface Science, 515, 146069, 2020.

CARVAlHO, G; ALMEIDA, Y; ALVES, K; FARIAS, J; SIMÕES, V; BRAGA, A. Avaliação estrutural e morfológica da hidroxiapatita sintetizada por pechini. Brazilian Journal of Development, 6, 71812 - 71821, 2020.

CHEN, Z; LIU, Y; MAO, L; GONG, L; SUN, W; FENG, L. Effect of cation doping on the structure of hydroxyapatite and the mechanism of defluoridation. Ceramics International, 44, 6, 6002-6009, 2018.

CIOBANU, G; HARJA, M. Cerium-doped hydroxyapatite/collagen coatings on titanium for bone implants. Ceramics International, 45, 2(B), 2852-2857, 2019.

DUBNIKA, A; ZALITE, V. Preparation and characterization of porous Ag doped hydroxyapatite bioceramic scaffolds. Ceramics International, 40, 7(A), 9923-9930, 2014.

EPPLE, M. Review of potential health risks associated with nanoscopic calcium phosphate. Acta Biomaterialia, 77, 1-14, 2018.

FARIAS, J; CARVALHO, G; ALVES, K; ALMEIDA, Y; PEREIRA, G; COSTA, S; BRAGA, A. Síntese de hidroxiapatita pelo método dos precursores poliméricos. Brazilian Journal of Development, 6, 76662 - 76670, 2020.

FATHI, M. H; HANIFI, A. Evaluation and characterization of nanostructure hydroxyapatite powder prepared by simple sol-gel method. Materials Letters, 61, 18, 3978-3983, 2007.

GOMES, L. C; DI LELLO, B. C; CAMPOS, J. B; SAMPAIO, M. Synthesis and characterization of calcium phosphates produced from chicken eggshell. Cerâmica 58, 448-452, 2012.

GUARIVO, V; IAFISCO, M; SPRIANO, S. 1 - Introducing biomaterials for tissue repair and regeneration. Nanostructured Biomaterials for Regenerative Medicine, Woodhead Publishing Series in Biomaterials 2020, Pages 1-27.

GUASTALDI, A. C; APARECIDA, A. H. Calcium phosphates of biological interest: importance as biomaterials, properties and methods for coatings obtaining. Quím. Nova, 33, 6 São Paulo, 
2010.

IBRAHIM, M; LABAKI, M; GIRAUDON, JM; LAMONIER, JF. Hydroxyapatite, a multifunctional material for air, water and soil pollution control: A review. Journal of Hazardous Materials, 383, $121139,2020$.

KAKIHANA, M. Sol-gel preparation of high temperature superconducting oxides. Journal of Sol-Gel Science and Technology, 6, 7 - 55, 1996.

KONERMANN, A; STAUBWASSER, M; DIRK, C; KEILIG, L; BOURAUEL, C; GOTZ, W; JAGER, A; REICHERT, C. Bone substitute material composition and morphology differentially modulate calcium and phosphate release through osteoclast-like cells. International Journal of Oral and Maxillofacial Surgery, 43 (4), 514-521, 2014.

KONG, W; ZHAO, K; GAO, C; ZHU, P. Synthesis and characterization of carbonated hydroxyapatite with layered structure. Materials Letters, 255, 126552, 2019.

KUMAR, S; NEHRA, M; KEDIA, D; DILBAGHI, N; TANKESHWAR, K; KIM, KH. Nanotechnology-based biomaterials for orthopaedic applications: Recent advances and future prospects. Materials Science and Engineering: C, 106, 110154, 2020.

KUMAR, V; SHARMA, R; KUMAR, S; KAUR, M; SHARMA, J. D. Enhancement in the photocatalytic activity of $\mathbf{B i}_{\mathbf{2}} \mathbf{T i}_{\mathbf{2}} \mathbf{O}_{7}$ nanopowders synthesised via Pechini vs Co-Precipitation method. Ceramics International, 45(16), 20386-20395, 2019.

LUO. W; HUANG, Y; ZHOU, X; HAN, Q; PENG, X; REN, B; LI, J; LI, M; CHENG, L. The effect of disaggregated nano-hydroxyapatite on oral biofilm in vitro. Dental Materials, 36, 7, e207-e216, 2020.

MAHMOOD, B. K; KAYGILI, O; BULUT, N; DOROZHKIN, S. V; ATES, T; KOYTEPE, S; GURSES, C; ERCAN, S; KEBIROGLU, H; AGID, R. S; INCE, T. Effects of strontium - erbium co-doping on the structural properties of hydroxyapatite: An Experimental and theoretical study. Ceramics International, 46, 10(B), 16354-16363, 2020.

MARTINEZ-GARCIA, N. O; ESPARZA-GONZALEZ，S. C; CASTILLO-MARTINEZ，N. A; SERRANO-MEDINA, A; OLIVAS-ARMENDARIZ, I; CAMPOS-MUZQUIZ, L. G; MUZQUIZRAMOS, E. M. Synergism in novel silver-copper/hydroxyapatite composites for increased antibacterial activity and biocompatibility. Ceramics International, 46, 12, 20215-20225, 2020.

MOHD PU'AD, N. A. S; KOSHY, P; ABDULLAH, H. Z; IDRIS, M. I; LEE, T. C. Syntheses of hydroxyapatite from natural sources. Heliyon, 5, 5, e01588, 2019.

MONTOYA-CISNEROS, K. L; RENDÓN-ANGELES, J. C; MATAMOROS-VELOZA, Z; MATAMOROS-VELOZA, A; YANAGISAWA, K. Low-temperature densification of Mgdoped hydroxyapatite fine powders under hydrothermal hot processing conditions. Ceramics 
International, 43, 15, 11907-11919, 2017.

NAIK, K. S. Chapter 25 - Advanced bioceramics. Advances in Biological Science Research, A Practical Approach, 411-417, 2019.

NGUYEN, D. K; BACH, QV; KIM, B; LEE, H; KANG, C; KIM, IT. Synthesis of Cr-doped Al $\mathbf{O}_{3}$ by Pechini sol-gel method and its application for reversible thermochromic sensors. Materials Chemistry and Physics, 223, 708-714, 2019.

OMORI, Y; OKADA, M; TAKEDA, S; MATSUMOTO, N. Fabrication of dispersible calcium phosphate nanocrystals via a modified Pechini method under non-stoichiometric conditions. Materials Science and Engineering: C, 42, 562-568, 2014.

PALUSZKIEWICZ, C; SLÓSARCZYK, A; PIJOCHA, D; SITARZ, M; BUCKO, M; ZIMA, A; CHROSCICKA, A; LEWANDOWSKA-SZUMIEL, M. Synthesis, structural properties and thermal stability of Mn-doped hydroxyapatite. Journal of Molecular Structure, 976, 1-3, 301-309, 2010.

PECHINI, M. P. Method of preparing lead and alkaline earth titanates and niobates and coating method using the same to form a capacitor. US Patent No. 3330697, 1967.

PEKOUNOV, Y; CHAKAROVA, K; HADJIVANOV, K. Surface acidity of calcium phosphate and calcium hydroxyapatite: FTIR spectroscopic study of low-temperature CO adsorption. Materials Science and Engineering: C, 29, 4, 1178-1181, 2009.

PEÑA, J; VALLET-REGI, M. Hydroxyapatite, tricalcium phosphate and biphasic materials prepared by a liquid mix technique. Journal of the European Ceramic Society, 23(10), 1687-1696, 2003.

PIERRE, C; BERTRAND, G; REY, C; BENHAMOU, O; COMBES, C. Calcium phosphate coatings elaborated by the soaking process on titanium dental implants: surface preparation, processing and physical-chemical characterization. Dental Materials, Volume 35 (2), 25-35, 2019.

PINA, S; REIS, R. L; OLIVEIRA, J. M. Ceramic biomaterials for tissue engineering. Fundamental Biomaterials: Ceramics, 95-116, 2018.

PUBBY, K; MEENA, S. S ; YUSUF, S. M; NARANG, S. B. Cobalt substituted nickel ferrites via Pechini's sol-gel citrate route: $\mathrm{X}$-band electromagnetic characterization. Journal of Magnetism and Magnetic Materials, 466, 430-445, 2018.

RAJABI-ZAMANI,A.H; BEHNAMGHADER,A; KAZEMZADEH,A. Synthesis of nanocrystalline carbonated hydroxyapatite powder via nonalkoxide sol-gel method. Materials Science and Engineering: C, 28, 8, 1326-1329, 2008. 
RAMIREZ, A. E; SOLARTE, N. J; SINGH, L. H; COAQUIRA, J. A. H; GAONA, S. Investigation of the magnetic properties of $\mathrm{SrFe}_{12} \mathrm{O}_{19}$ synthesized by the Pechini and combustion methods. Journal of Magnetism and Magnetic Materials, 438, 100-106, 2017.

RAVAL, N; KALYANE, D; MAHESHWARI, R; TEKADE, R. K. Chapter 17 - Surface Modifications of Biomaterials and Their Implication on Biocompatibility. Biomaterials and Bionanotechnology Advances in Pharmaceutical Product Development and Research, 639-674, 2019.

RIAZ, M; ZIA, R; IJAZ, A; HUSSAIN, T; MOHSIN, M; MALIK, A. Synthesis of monophasic Ag doped hydroxyapatite and evaluation of antibacterial activity. Materials Science and Engineering: C, 90, 308-313, 2018.

RUSTOM, L. E; POELLMAN, M. J; JOHNSON, A. J. W. Mineralization in micropores of calcium phosphate scaffolds. Acta Biomaterialia, 83, 435-455, 2019.

SANTHIYAVIMAL, S; VASANTHARAJ, S; LEWISOSCAR, L; SELVARAJ, R; BRINDHADEVI, K; PUGAZHENDHI, A. Natural organic and inorganic-hydroxyapatite biopolymer composite for biomedical applications. Progress in Organic Coatings, 147, 105858, 2020.

SANTOS, M. V. B; OLIVEIRA, A. L; OSAJIMA, J. A; FIHO, E. C. S. Development of composites scaffolds with calcium and cerium-hydroxyapatite and gellan gum. Ceramics International, 46, 3, 811-3817, 2020.

SANTOS, T. V; PRYSTON, D. B. A; ASSIS, G. C; MENEGHETTI, M. R; MENEGHETTI, S. M. $\mathrm{P}$. Tin, niobium and tin-niobium oxides obtained by the Pechini method using glycerol as a polyol: synthesis, characterization and use as a catalyst in fructose conversion. Catalysis Today, Available online 23 July 2020.

SENRA, M. R; LIMA, R. B; SOUZA, D. H. S; MARQUES, M. F. V; MONTEIRO, S. N. Thermal characterization of hydroxyapatite or carbonated hydroxyapatite hybrid composites with distinguished collagens for bone graft. Journal of Materials Research and Technology, 9, 4, 71907200,2020 .

SHALY, A. A; PRIYA, G. H; LINET, J. M. An outlook on the mechanical attributes and load curve analysis of hydrothermally acquired hydroxyapatite bioceramic nanoparticles. Physica B: Condensed Matter, 590, 412223, 2020.

SHYONG, Y-J; CHANG, K-C; LIN, F-H. Calcium phosphate particles stimulate exosome secretion from phagocytes for the enhancement of drug delivery. Colloids and Surfaces B: Biointerfaces, 171, 391-397, 2018.

STARTEK, K; MARCZAK, J; LUKOWIAK, A. Oxygen barrier enhancement of polymeric foil by sol-gel-derived hybrid silica layers. Polymer, 195, 122437, 2020. 
SUPOVÁ, M. Substituted hydroxyapatites for biomedical applications: A review. Ceramics International, 41, 8, 9203-9231, 2015.

SWAMIAPPAN, S. Synthesis of carbonate substituted hydroxyapatite by Pechini method. Kuwait Journal of Science, 43 (1) pp. 174-184, 2016.

TREDWIN, C. J; YOUNG, A. M; GEORGIOU, G; SHIN, SH; KIM, HW, KNOWLES, J. C. Hydroxyapatite, fluor-hydroxyapatite and fluorapatite produced via the sol-gel method. Optimisation, characterisation and rheology. Dental Materials, 29, 2, 166-173, 2013.

ULLAH, I; SIDDIQUI, M. A; KOLAWOLE, S. K; LIU, H; ZHANG, J; REN, L; YANG, K. Synthesis, characterization and in vitro evaluation of zinc and strontium binary doped hydroxyapatite for biomedical application. Ceramics International, 46, 10(A), 14448-14459, 2020.

UNABIA, R. B; BONEBEAU, S; CANDIDATO JR; R. T; PAWLOWSKI, L. Preliminary study on copper-doped hydroxyapatite coatings obtained using solution precursor plasma spray process. Surface and Coatings Technology, 353, 370-377, 2018.

VAHDAT, A; GHASEMI, B; YOUSEFPOUR, M. Mechanical properties of the hydroxyapatite and magnetic nanocomposite of hydroxyapatite adsorbents. South African Journal of Chemical Engineering, 33, 90-94, 2020.

VALLET-REGÍ, M; BALAS, F; COLILLA, M; MANZANO, M. Bone-regenerative bioceramic implants with drug and protein controlled delivery capability. Progress in Solid State Chemistry, 36, 3, 163-191, 2008.

WANG, C; JEONG, K-J; KIM, J; KANG, S. W; KANG, J; HAN, I. H; LEE, IW; OH, SJ; LEE, J. Emission-tunable probes using terbium (III)-doped self-activated luminescent hydroxyapatite for in vitro bioimaging. Journal of Colloid and Interface Science, Available online 21 July 2020.

WANG, YT; ZHANG, XT; XU, JB; SHEN, X; WANG, CA; LI, FW; ZHANG, ZH; CHEN J; YE, $\mathrm{YH}$; SHEN, RQ. Fabrication and characterization of Al-CuO nanocomposites prepared by solgel method. Defence Technology, Available online 14 July 2020.

WINCK, L. B; FERREIRA, J. L. A; MARTINEZ, J. M. G; ARAUJO, J. A; RODRIGUES, A. C. M; SILVA, C. R. M. Synthesis, sintering and characterization of ceria-based solid electrolytes codoped with samaria and gadolinium using the Pechini method. Ceramics International, 43, 18, 16408-16415, 2017.

WU, S; MA. S; ZHANG, C; CAO, G; WU, D; GAO, C; LAKSHMANAN, S. Cryogel biocomposite containing chitosan-gelatin/cerium-zinc doped hydroxyapatite for bone tissue engineering. Saudi Journal of Biological Sciences, Available online 1 June 2020.

YADAV, S; SINGH,P; PYARE, R. Synthesis, characterization, mechanical and biological properties of biocomposite based on zirconia containing 1393 bioactive glass with hydroxyapatite. Ceramics International, 46, 8(A), 10442-1045, 2020. 
ZHANG, W; CAO, N; CHAI, Y; XU, X; WANG, Y. Synthesis of nanosize single-crystal strontium hydroxyapatite via a simple sol-gel method. Ceramics International, 40, 10(A), 16061-16064, 2014. 


\section{Índice Remissivo}

A

Ácido cítrico 11, 12, 13, 14, 15, 16, 19, 20, 22, 23, 25, 35, 37, 39, 41, 45, 51, 52, 53

Ácido cítrico/cátions metálicos 11, 12, 13, 14, 16, 25, 35, 37, 45, 52, 53

Água residual 11, 15, 23

Álcool etílico 11, 12, 27, 28, 29, 30, 31, 53

Álcool isopropílico 27, 28, 30, 31

Alumínio 12, 13, 14, 20, 27, 28, 30, 31

Ambiente fisiológicos 45

Ambientes químicos 12, 19

Análise térmica 11, 12, 19, 21, 27, 29

Aplicações tecnológicas 11, 20, 68

Assistência à saúde humana 35

\section{B}

Beta fosfato tricálcico ( $\beta$-tcp) 35,39

Bioatividade 35, 45, 47, 49

Biocerâmicas 35, 45, 46, 47

Biocompatibilidade 37, 45, 46, 47, 49

Biomateriais 45, 46, 47, 50

Biomaterial cerâmico 35

$\mathrm{C}$

Calcinação 12, 19, 20, 21, 24, 27, 29, 30, 31, 37, 51

Cátions metálicos 11, 13, 14, 16, 19, 20, 35, 39, 41, 52

Cerâmicas perovskitas 61

Ciência/indústria 61,63

Condutividade térmica 12, 19

Cristalização da mulita 11, 14, 16, 19, 21, 24, 27, 31

$\mathrm{D}$

Decomposição dos componentes 11, 15, 23

Densidade 12, 19, 48, 63

Difração de raios x (drx) 11, 27, 29, 35, 38

E

Engenharia de tecidos 35, 36

Escala de homogeneidade 27

Estabilidade 12, 19, 35, 46, 50, 53, 62

Estabilidade térmica 12, 19, 46, 53

Estrôncio 49, 61, 62, 63, 65, 66, 67, 68, 70

Expansão térmica 12,19

F 
Fase alfa-alumina 19

Fase alumina 11

Fase mulita 11, 19, 22, 29, 30

Fases cristalinas 19

Fases de fosfatos de cálcio 35

Fosfato de cálcio bifásico 35

G

Géis difásicos 27

Géis monofásicos 27

$\mathrm{H}$

Hidroxiapatita (hap) 35

Hipertermia magnética 61, 63

$\mathrm{L}$

Liberador de fármacos 61,63

M

Manganita de lantânio 61, 62, 63, 70

Material cerâmico 11

Material orgânico 11, 15, 23

Mecanismos de reação 19

Método pechini $11,12,13,14,15,16,17,19,20,24,25,37,39,41,45,47,51,52,53$

Método sol-gel 20, 27, 28, 31, 37

Microambiente fisiológico 35

Microscopia eletrônica de varredura (mev) 19, 21, 35, 38

Mulita 11, 19, 20, 27, 28

$\mathrm{N}$

Nanofibras 61

Nano/micrométricos e monólitos 61

Nanotecnologia 61, 63

Nível atômico 13, 27

$\mathrm{O}$

Organismo humano 45

Osseointegração 35,37

$\mathrm{P}$

Perda ou falha de tecidos ou órgãos 35

Processamento sol-gel 27

$\mathrm{R}$

Refratariedade e desempenho do material 19

Resistência mecânica $12,19,46$ 
Ressonância magnética 61, 63

$\mathrm{S}$

Silício 13, 14, 27, 31

Síntese 11, 12, 13, 14, 19, 20, 25, 27, 28, 37, 39, 45, 47, 50, 51, 52, 53, 63

Solvente 11, 19, 20, 21, 22, 24, 27, 28, 30, 31, 45, 53

$\mathrm{T}$

Tetrahidrofurano 19

Tipos de géis 27

Toxicidade $37,38,40,41,42,45,47$ 
EDITORA

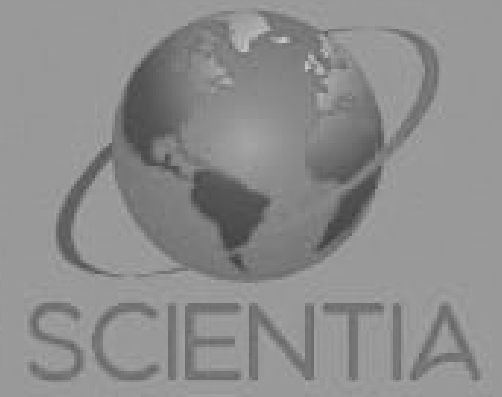

OMNIS SCIENTIA

editoraomnisscientia@gmail.com M https://editoraomnisscientia.com.br/ @editora_omnis_scientia (0) https://www.facebook.com/omnis.scientia.9 $\subsetneq$

+55 (87) 9656-3565 @ 
EDITORA

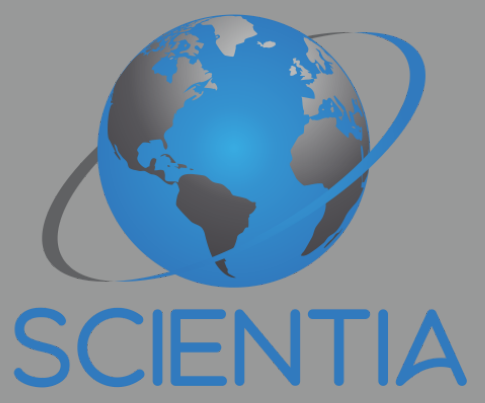

OMNIS SCIENTIA

editoraomnisscientia@gmail.com M https://editoraomnisscientia.com.br/ ( @editora_omnis_scientia (0) https://www.facebook.com/omnis.scientia.9 $\subsetneq$ +55 (87) 9656-3565 @ 